\title{
Risk factors for bleeding complications in patients undergoing transcatheter aortic valve implantation (TAVI)
}

Janina Stępińska ${ }^{1}$, Katarzyna Czerwińska ${ }^{2}$, Adam Witkowski ${ }^{3}$, Maciej Dąbrowski ${ }^{3}$, Zbigniew Chmielak ${ }^{3}$, Krzysztof Kuśmierski ${ }^{4}$, Tomasz Hryniewiecki ${ }^{2}$, Marcin Demkow ${ }^{5}$

${ }^{1}$ Intensive Cardiac Care Clinic, Institute of Cardiology, Warsaw, Poland

${ }^{2}$ Department of Acquired Cardiac Defects, Institute of Cardiology, Warsaw, Poland

${ }^{3}$ Department of Cardiology and Interventional Angiology, Institute of Cardiology, Warsaw, Poland

${ }^{4}$ Department of Cardiac Surgery and Transplantology, Institute of Cardiology, Warsaw, Poland

${ }^{5}$ Department of Coronary Artery Disease and Structural Heart Disease,

Institute of Cardiology, Warsaw, Poland

\begin{abstract}
Background: The risk of bleedings in transcatheter aortic valve implantation (TAVI) patients increases due to age and concomitant diseases. The aim of the study was to assess the risk of bleedings, their influence on early prognosis of TAVI patients and utility of the TIMI and GUSTO scales in the evaluation of bleeding and in prediction of blood transfusion.

Methods: This was a single center study of in-hospital bleedings in 56 consecutive TAVI patients. Bleedings were classified according to the GUSTO and TIMI scales. HASBLED's scale risk factors, diabetes mellitus, female sex, the route of bioprosthesis implantation and inhospital antithrombotic treatment were analyzed. Statistical analysis consisted of $\chi^{2}$, Fisher's exact, Wilcoxon tests and logistic regression analysis.
\end{abstract}

Results: Serious bleedings occurred in 35 (62.5\%) patients. There was no significant correlation with HASBLED score. History of anemia was a significant predictor of bleeding in GUSTO $(p=0.0013)$ and TIMI $(p=0.048)$ scales. No bleedings in patients receiving vitamin K antagonists (VKA) pre- and VKA plus clopidogrel post intervention were observed. Patients with bleedings according to the GUSTO scale more often required blood tranfusion than in TIMI scale ( $p=0.03)$.

Conclusions: History of anemia is the strongest predictor of serious bleedings. VKA before and VKA with clopidogrel after TAVI are safer than dual antiplatelet or triple therapy. The TIMI and GUSTO scales can adequately classify bleeding after TAVI, however the GUSTO better predicts transfusions. (Cardiol J 2013; 20, 2: 125-133)

Key words: transcatheter aortic valve implantation (TAVI), bleeding complications, aortic stenosis, antithrombotic therapy

Address for correspondence: Prof. Janina Stępińska, Intensive Cardiac Care Clinic, Institute of Cardiology, ul. Alpejska 42, 04-628 Warszawa, Poland, tel: +48 2234343 14, fax: +48 2281542 67, e-mail: janina@stepinska.pl.pl 


\section{Introduction}

The rules of antithrombotic treatment after surgical aortic valve replacement are known [1]. It is accepted that patients after thranscatheter aortic valve implantation (TAVI) receive dual antiplatelet therapy (DAPT) with acetylsalicylic acid and clopidogrel in prevention of thrombo-embolic complications [2]. Until now there have not been any prospective studies assessing the efficacy and safety of this type of treatment. The risk of bleeding complications in population of patients undergoing TAVI is increased due to age of the patients and the presence of numerous concomitant diseases. Bleeding complications and blood transfusions worsen the early and long-term prognosis of patients after interventions [3-6].

The aim of the study was to assess the risk of bleeding complications and their influence on early prognosis of patients undergoing TAVI. Additional aim was to analyze the utility of the TIMI [7] and GUSTO [8] bleeding scales in the evaluation of bleeding complications and the usefulness of those scales in prediction of blood transfusion necessity in the early post-procedural period.

\section{Methods}

The study included 56 consecutive patients between 62 and 91 years of age $(81.89 \pm 6.12)$ who underwent TAVI in the Institute of Cardiology in Warsaw between January 2009 and February 2011.

This was a single center, observational study of early, in-hospital bleeding complications related to TAVI, retrospective until February 2011 and from that point on prospective. Analyzed parameters were: (1) risk factors of bleeding complications in the HASBLED scale: hypertension, renal failure, liver failure, previous stroke/transient ischemic attack (TIA), previous bleeding, history of anemia, nontherapeutic international normalized ratio (INR), age, use of antiplatelet drugs or nonsteriodal anti-inflamatory drugs, alcohol abuse, (2) diabetes mellitus type 2, (3) female sex, (4) the route of bioprosthesis implantation - transarterial or transapical and (5) pre- and post-procedural antithrombotic treatment. Although in 2011 Valve Academic Research Consotrium (VARC) had already created a scale dedicated for TAVI bleedings [9], we used two known bleeding scales: TIMI [7] and GUSTO [8] for bleeding classification. We decided to compare their utility in bleedings classification and predictory value in the assessment of blood transfusion necessity. Major bleeding according to TIMI scale was defined as intracranial hemorrhage or $\mathrm{a} \geq 5 \mathrm{~g} / \mathrm{dL}$ decrease in the hemoglobin concentration (HGBc) or $\mathrm{a} \geq 15 \%$ absolute decrease in the hematocrit (HCT). Minor bleeding according to TIMI scale was defined as observed blood loss with $\geq 3 \mathrm{~g} / \mathrm{dL}$ decrease in the HGBc or $\geq 10 \%$ decrease in the HCT, or non observed blood loss with $\geq 4 \mathrm{~g} / \mathrm{dL}$ decrease in the $\mathrm{HGBc}$ or $\geq 12 \%$ decrease in the HCT. Minimal TIMI bleeding was defined as any clinically overt sign of hemorrhage (including imaging) that is associated with $\mathrm{a}<3 \mathrm{~g} / \mathrm{dL}$ decrease in the HGBc or $<9 \%$ decrease in the HCT. Severe or life-threatening bleeding according to GUSTO scale was defined as either intracranial hemorrhage or bleeding that causes hemodynamic compromise and requires intervention. Moderate bleeding according to GUSTO scale was deifined as bleeding that requires blood transfusion but does not result in hemodynamic compromise. Mild GUSTO bleeding was defined as bleeding that does not meet criteria for either severe or moderate bleeding.

The history of anemia was defined as HGBc $<12 \mathrm{~g} / \mathrm{dL}$, diagnosed on the basis of blood count a day before the procedure. The decrease in $\mathrm{HGBc}$ and HCT after TAVI was assessed on the basis of blood count, collected every day during 2 consecutive days and fiveth day after the procedure, which was compared with HGBc and HCT value on a day before the procedure. The transfusion was made when the post-TAVI HGBc was $\leq 10.0 \mathrm{~g} / \mathrm{dL}$, HCT $\leq 30.5 \%$ and decrease in $\mathrm{HGBc}$ was $\geq 1.5 \mathrm{~g} / \mathrm{dL}$, in $\mathrm{HCT} \geq 5 \%$, or when the post-procedural HGBc was $\leq 9.5 \mathrm{~g} / \mathrm{dL}$, or HCT $\leq 28 \%$ and patient had periprocedural vascular complications which could possibly lead to further decline in HGBc or HCT. The decision of the the number of transfused pocket red blood cells units (PRBCu) was based on the individual physiscian evaluation.

Vascular complications were defined as injury of the vessels (femoral artery, iliac artery, aorta) directly related with the bioprosthesis implantation, access site or acess access-related vascular injury (dissection, stenosis, perforation, rupture, arteriovenous fistula, pseudoaneurysm, haematoma), also pleural hemathoma or haemorhage during transapical aortic-valve implantation (TA-AVI), which can lead to death, need for blood transfusions and/or unplanned percutaneous or surgical intervention or requiring compression or thrombin injection therapy in the case of pseudoaneurysm.

All patients undergoing the procedure had at least mild bleeding and due to the small number of the paricipants, in all statistical analyzes the moderate/minor and severe/major TIMI/GUSTO bleedings were assessed together as serious bleedings vs. mild/minimal incidents. The studied group was di- 
vided into two subgroups to assess the influence of a learning curve on cardiovascular complications.

Severe aortic valve stenosis was defined according to the European Society of Cardiology (ESC) criteria on the diagnosis and management of valve diseases published in 2007 [1]. Patients were qualified for the TAVI procedure according to ESC/ /European Association of Cardio-Thoracic Surgeons (EACTS) criteria prepared in collaboration with European Association on Percutaneous Coronary Interventions (EAPCI) in 2008 [2]. Two types of protheses were used: Edwards-Sapien or Sapien XT (ES) bioprosthesis (Edwards Lifesciences) of 23 and $26 \mathrm{~mm}$ diameter with 18 and 22-French vascular sheath systems and CoreValve (CV) bioprosthesis (CoreValve Revalving System, Medtronic) of 26 and $29 \mathrm{~mm}$ diameter with the system of 18 French delivery cetheters. The type of bioprosthesis and the route of implantation were adjusted to the size of the native valve annulus and to the diameter and status of peripheral arteries. The TAVI procedure was performed under aseptic conditions in catheterization laboratory under general anesthesia and guided with transesophageal echocardiography. For the CV prosthesis a transfemoral or transsubclavian access were used and for the ES prosthesis a transfemoral or transapical access were applied depending on the anatomic conditions. All patients received unfractionated heparin during the procedure under activated partial thromboplastin time control.

The study was approved by the local research ethics committee and all patients in prospective part of the study were asked for written informed consent.

\section{Statistical analysis}

Univariate and multivariate statistical analysis was performed with means of the SAS system. Categorical data are presented as frequencies and percentages; continuous variables are expressed as means \pm SD. Comparisons were made with the $\chi^{2}$ statistic test or, when appropriate, the Fisher exact test for categorical variables and with the nonparametric Wilcoxon signed rank test for continuous variables. Uni- and multivariate logistic regression model with stepwise method selection of significance with odds ratios (ORs) and $95 \%$ confidence interval (CI) was performed to identify independent variables predicting the risk of in-hospital serious bleedings. Because of the small number of participants on various drugs combinations we did not perform multivariate analysis of peri-procedural treatment. For all statistical tests, a significance level of $\mathrm{p}<0.05$ was used.
Table 1. Baseline characteristics of the 56 patients.

\begin{tabular}{|c|c|}
\hline Age [year] & $81.89 \pm 6.13$ \\
\hline Female sex & $36(64.28 \%)$ \\
\hline Logistic EuroSCORE [\%] & $\begin{array}{c}6.59-59.1 \\
(27.69 \pm 13.00)\end{array}$ \\
\hline \multicolumn{2}{|l|}{ Implantation: } \\
\hline TF-AVI/TSC-AVI & $38 / 4(67.85 \% / 7.14 \%)$ \\
\hline TA-AVI & $14(25 \%)$ \\
\hline \multicolumn{2}{|l|}{ NYHA class: } \\
\hline II & $3(5.35 \%)$ \\
\hline III & $39(69.64 \%)$ \\
\hline IV & $14(25 \%)$ \\
\hline Coronary artery disease & $45(80.35 \%)$ \\
\hline Previous myocardial infarction & $15(26.78 \%)$ \\
\hline \multicolumn{2}{|l|}{ Previous intervention: } \\
\hline $\mathrm{PCl}$ & $21(37.5 \%)$ \\
\hline CABG & $9(16.07 \%)$ \\
\hline COPD & $20(35.71 \%)$ \\
\hline Atrial fibrillation & $23(41.07 \%)$ \\
\hline Permanent pacemaker: & $12 / 25$ \\
\hline pre/post TAVI & $(21.42 \% / 44.64 \%)$ \\
\hline Pulmonary hypertension & $37(66.07 \%)$ \\
\hline Extensively calcified aorta & $3(5.35 \%)$ \\
\hline Osteoporosis & $16(28.57 \%)$ \\
\hline BMI $\left[\mathrm{kg} / \mathrm{m}^{2}\right]$ & $25.32 \pm 3.21$ \\
\hline \multicolumn{2}{|l|}{ Echocardiographic data: } \\
\hline AVA $\left[\mathrm{cm}^{2}\right]$ & $0.65 \pm 0.11$ \\
\hline Aortic PG $\max [\mathrm{mm} \mathrm{Hg}]$ & $100 \pm 22.71$ \\
\hline Aortic PG med (mm Hg] & $60.78 \pm 17.09$ \\
\hline LVEF [\%] & $51.94 \pm 13.65$ \\
\hline Ejection fraction $<50 \%$ & $24(42.10 \%)$ \\
\hline \multicolumn{2}{|c|}{$\begin{array}{l}\text { TF-AVI - transfemoral aortic valve implantation; TSC-AVI - trans- } \\
\text { subclavian aortic valve implantation; TAA-AVI - transapical aortic } \\
\text { valve implantation; NYHA - New York Heart Association class of } \\
\text { heart failure; PCI - precutaneous coronary intervention; CABG - } \\
\text { coronary-artery bypass grafting; COPD - chronic obstructive pul- } \\
\text { monary disease; BMI - body mass index; AVA - aortic valve area; } \\
\text { PG - pressure gradient; LVEF - left ventricular ejection fraction }\end{array}$} \\
\hline
\end{tabular}

\section{Results}

The analysis included a group of 56 consecutive patients with severe, symptomatic aortic valve stenosis disqualified from surgical aortic valve replacemnet due to high procedural risk and/or lack of technical possibilities to perform the procedure. Detailed characteristics of patients undergoing TAVI is presented in Table 1.

Bleeding in the early post-procedural period occurred in all patients who underwent TAVI. Moderate and severe bleeding complications were noted in $35(62.5 \%)$ patients: $29(51.78 \%)$ patients fulfilled 
Table 2. Bleeding according to the GUSTO and TIMI scales and blood transfusion after TAVI in 56 patients.

\begin{tabular}{|c|c|c|c|c|c|c|}
\hline \multirow{2}{*}{$\begin{array}{l}\text { Bleeding } \\
\text { classifications }\end{array}$} & \multicolumn{3}{|c|}{ GUSTO } & \multicolumn{3}{|c|}{ TIMI } \\
\hline & $\begin{array}{c}\text { Bleeding } \\
\text { no. } / \%\end{array}$ & $\begin{array}{c}\text { Transfusion } \\
\text { pts. no. } \\
\text { (PRBCu/pt) }\end{array}$ & $\begin{array}{c}\text { PRBCu } \\
\text { min.-max. }\end{array}$ & $\begin{array}{l}\text { Bleeding } \\
\text { no. } / \%\end{array}$ & $\begin{array}{c}\text { Transfusion } \\
\text { pts. no. } \\
\text { (PRBCu/pt) }\end{array}$ & $\begin{array}{c}\text { PRBCu } \\
\text { min.-max. }\end{array}$ \\
\hline Mild/minimal & $26 / 46.42 \%$ & $2(3)$ & $0-2(0.05 \pm 0.29)$ & $37 / 66.07 \%$ & $17(44)$ & $0-5(0.78 \pm 1.37)$ \\
\hline Moderate/minor & $21 / 37.5 \%$ & $21(2.28)$ & $1-4(0.85 \pm 1.27)$ & $14 / 25.0 \%$ & $11(3.09)$ & $0-16(0.64 \pm 2.25)$ \\
\hline Severe/major & $8 / 14.28 \%$ & $8(7.0)$ & $1-17(1.07 \pm 3.38)$ & $5 / 8.92 \%$ & $3(9.66)$ & $0-17(0.55 \pm 2.60)$ \\
\hline Serious ${ }^{*}$ & $29 / 51.78 \%$ & $29(3.58)$ & $1-17(1.92 \pm 3.35)$ & $19 / 33.92 \%$ & $14(4.5)$ & $0-17(1.19 \pm 3.33)$ \\
\hline
\end{tabular}

${ }^{*}(p=0.03)$ : incidents of blood transfusions in serious GUSTO bleedings vs. serious TIMI bleedings; pts. no. — patients number; PRBCu — pocket red blood cells units; PRBCu/pt — pocket red blood cells units per patient

the GUSTO scale criteria and 19 (33.92\%) patients met the TIMI scale criteria; 33 of the 35 severe bleeding complications $(94.28 \%)$ were related to the route of bioprosthesis implantation. There were 17 vascular complications among 28 patients who underwent TAVI earlier in the study in comparison to 16 complications among 28 patients who underwent the procedure later in the study. Learning curve did not influence vascular complications related to serious bleeding ( $\mathrm{p}=\mathrm{NS}$ ). The frequency of bleeding according to the GUSTO and TIMI scales and the number of transfused PRBCu are presented in Table 2. The peri-procedural HGBc and HCT value are presented in Table 3 . The type of bleeding complications in the early post-procedural phase and their influence on in-hospital mortality is summarized in Table 4 .

The prevalence of analyzed risk factors in 56 patients and their relation to bleeding defined by the GUSTO and TIMI scales are presented in Table 5.

Univariate analysis demonstrated that the only predictor of serious bleeding complications according to the GUSTO and TIMI scales is the history of anemia ( $\mathrm{p}=0.0013$ and $\mathrm{p}=0.048$, respectively). Univariate logistic regression analysis showed that history of anemia is predictor of serious bleeding only according to GUSTO scale.

The influence of pre-procedural pharmacotherapy on bleeding after TAVI was analyzed. 52 (92.85\%) patients were on antithrombotic treatment before TAVI. No bleeding complications after intervention were observed in patients on vitamin $\mathrm{K}$ antagonist (VKA) monotherapy prepared for the procedure as recommended. All other patients suffered from bleeding complications. The risk of bleeding according to the GUSTO or TIMI scales in every subgroup in relation to VKA was compared (Table 6).

Subsequently the analysis focused on the influence of antithrombotic treatment after TAVI on early bleeding complications. One patient who died after few hours after the procedure due to disruption of the arotic wall and left ventricle during prosthesis implantation was excluded from the treatment analysis. Three patients were maintained on clopidogrel monotherapy due to high risk of bleeding complications. All other patients received a combined therapy. Bleeding was not observed only in patients treated after TAVI with VKA and clopidogrel. Safety of treatment with VKA plus clopidogrel in comparsion to other drugs used is presented in Table 7 .

There is no scale to assess the risk of bleeding complications among patients undergoing TAVI. The HASBLED scale constructed for patients with atrial fibrillation (AF) chronically treated with antithrombotic drugs was used for the purpose of the presented analysis. Almost all patients in the studied group (52 patients, $92.85 \%$ ) had a high risk of bleeding in the HASBLED scale ( $\geq 3$ points). Low risk was observed in only 4 patients of whom 3 suffered from bleeding complications defined as major in the TIMI scale and 2 had bleeding complications defined as severe in the GUSTO scale. There was no significant correlation between the HASBLED score and the number of patients with serious bleeding according to the TIMI and GUSTO scale ( $p=0.07$ and $p=0.88$, resepectively). Blood transfusion was necessary in all patients $29(100 \%)$ patients with serious GUSTO bleeding. They received a total number of $104 \mathrm{PRBCu}$ and 3.58 PRBCu per patient. Analogically, blood transfusion was necessary in 14 of the $19(73.98 \%)$ patients with serious TIMI bleeding; a total number of $63 \mathrm{PRBCu}$ was transfused, 4.5 $\mathrm{PRBCu}$ per patient (Table 2). $\mathrm{Pa}$ tients with serious GUSTO bleeding required blood transfusion more frequently than patients with serious TIMI bleeding $(\mathrm{p}=0.03)$.

There were 4 deaths during hospitalization. Three of them were caused by bleeding complications: 
Table 4. The causation of moderate and severe bleeding complications in early post-procedural phase and their influence on in-hospital mortality.

\begin{tabular}{lcc}
\hline $\begin{array}{l}\text { In hospital bleeding } \\
\text { after TAVI }\end{array}$ & No. $(\%)$ & Death no. \\
\hline $\begin{array}{l}\text { Vascular complications: } \\
\text { TF-AVI/TSC-AVI }\end{array}$ & $24(42.85 \%)$ & 2 \\
$\begin{array}{l}\text { TA-AVI (pleural } \\
\text { haemorrhage) }\end{array}$ & $9(16.07 \%)$ & 0 \\
$\begin{array}{l}\text { Gastrointerstinal } \\
\text { haemorrhage }\end{array}$ & $1(1.78 \%)$ & 1 \\
$\begin{array}{l}\text { Pericardial tamponade } \\
\text { Without bleeding }\end{array}$ & $1(1.78 \%)$ & 0 \\
Total & $21(37.5 \%)$ & 1 \\
\hline
\end{tabular}

TF-AVI - transfemoral aortic valve implantation; TSC-AVI — transsubclavian aortic valve implantation; TA-AVI — transapical aortic valve implantation

2 related to the vascular complications and 1 caused by massive bleeding from the upper gastrointestinal tract. A fourth death which occurred in the distant day of hospitalization was caused by decompensated heart failure. Serious bleeding did not have impact on in-hospital mortality $(\mathrm{p}=0.29)$, irrespective of the GUSTO and TIMI classification $(\mathrm{p}=0.37$ and $\mathrm{p}=0.07$, respectively), similarly as incidents od blood transfusions $(\mathrm{p}=0.41)$ and $\mathrm{PRBCu}(\mathrm{p}=0.28)$.

None of analized factors for TIMI bleeding entered into the multivariate model, because of its insignificance in univariate regression analysis. In stepwise regression analysis of GUSTO risk factors only anemia was significant predictor of in-hospital serious bleeding (OR 6.750; 95\% CI 1.946-23.416; $\mathrm{p}=0.0026)$.

\section{Discussion}

Presented population is typical for TAVI. Mean logistic Euroscore value was $27.69 \pm 13.00 \%$. Few patients were qualified for TAVI despite low Euroscore values due to the presence of risk factors not included in the prognostic scales such as porcelain aorta or diffused osteoporosis. Low Euroscore or STS values in these patients demonstrate that those scales are suboptimal and for that reason construction of new scales has been proposed [10, 11]. Most frequently described early, in-hospital complications of TAVI include: vascular complications, bleeding, stroke and TIA, death, the need of pacemaker implantation and acute renal failure [12-18]. In our study serious bleeding complications were observed in $35(62.5 \%)$ patients. Almost all of the early bleeding complications 33 (94.3\%) were re- 
Table 5. The prevalence and predictive value of bleeding risk factors on serious bleeding according to the GUSTO and TIMI scales in $\chi^{2}$ and univariate logistic regression analysis in 56 patients.

\begin{tabular}{|c|c|c|c|}
\hline Variables & $\begin{array}{c}\text { GUSTO bleeding } \\
\text { Yes }(n=29) / \text { No }(n=27)\end{array}$ & $\mathbf{P}$ & OR $(95 \% \mathrm{Cl}) ; \mathrm{p}$ \\
\hline Hypertension & $21(72.41 \%) / 13(48.14 \%)$ & 0.3 & $0.457(0.120-1.740) ; 0,25$ \\
\hline Abnormal renal function* & $17(58.62 \%) / 15(55.55 \%)$ & 0.64 & $1.133(0.393-3.269) ; 0.81$ \\
\hline Abnormal liver function $^{\dagger}$ & $1(3.44 \%) / 2(7,4 \%)$ & 0.47 & $0.446(0.038-5.227) ; 0.52$ \\
\hline History of stroke/TIA & $2(6.89 \%) / 4(14.81 \%)$ & 0.29 & $0.326(0.058-1.845) ; 0.20$ \\
\hline History of bleeding & $2(6.89 \%) / 4(14.81 \%)$ & 0.29 & $0.426(0.071-2.541) ; 0.34$ \\
\hline Anemia $^{\ddagger}$ & $21(72.41 \%) / 7(25,92 \%)$ & 0.0013 & $7.500(2.293-24.527) ; 0.0009$ \\
\hline Labile INRs & $0(0 \%) / 2(7.4 \%)$ & $0.12^{* *}$ & $* * *$ \\
\hline Age [year] & $83.35 \pm 5.88 / 80.33 \pm 6.11$ & 0.16 & $1.092(0.991-1.204) ; 0.07$ \\
\hline $\begin{array}{l}\text { Drugs (antiplatelet agents/ } \\
\text { /NSAIDs) }{ }^{5} / \text { Alkohol abuse }\end{array}$ & $2(6.89 \%) 0(0 \%)$ & $0.49^{* *}$ & $* * *$ \\
\hline Diabetes mellitus & $10(34.48 \%) / 14(51.85 \%)$ & 0.31 & $0.489(0.167-1.432) ; 0.19$ \\
\hline Female sex & $20(68.96 \%) / 16(59.25 \%)$ & 0.45 & $1.528(0.509-4.586) ; 0.44$ \\
\hline \multicolumn{4}{|l|}{ Implantation: } \\
\hline TF-AVI+TSC-AVI & $19(65.51 \%) / 23(85.18 \%)$ & 0.3 & $3.026(0.817-11.206) ; 0.09$ \\
\hline TA-AVI & $10(34.48 \%) / 4(14.81 \%)$ & & \\
\hline Variables & $\begin{array}{c}\text { TIMI bleeding } \\
\text { Yes }(n=19) / \text { No }(n=37)\end{array}$ & $\mathbf{P}$ & OR $(95 \% \mathrm{Cl}) ; \mathrm{p}$ \\
\hline Hypertension & $13(68.42 \%) / 21(56.75 \%)$ & 0.18 & $0.419(0.114-1.545) ; 0.19$ \\
\hline Abnormal renal function ${ }^{*}$ & $10(52.63 \%) / 22(59.45 \%)$ & 0.62 & $0.758(0.249-2.309) ; 0.62$ \\
\hline Abnormal liver function ${ }^{\dagger}$ & $1(5.26 \%) / 2(5.40 \%)$ & 0.96 & $0.972(0.083-11.459) ; 0.98$ \\
\hline History of stroke/TIA & $3(15.78 \%) / 3(8.1 \%)$ & 0.37 & $1.547(0.309-7.752) ; 0.59$ \\
\hline History of bleeding & $1(5.26 \%) / 5(13.51 \%)$ & 0.34 & $0.356(0.038-3.285) ; 0.36$ \\
\hline Anemia $^{\ddagger}$ & $6(31.57 \%) / 22(59.45 \%)$ & 0.048 & $0.315(0.098-1.013) ; 0.52$ \\
\hline Labile INRs & $0(0 \%) / 2(5.40 \%)$ & $0.54^{* *}$ & $* * *$ \\
\hline Age [year] & $82.32 \pm 4.99 / 81.68 \pm 6.69$ & 0.62 & $1.018(0.927-1.117) ; 0.70$ \\
\hline $\begin{array}{l}\text { Drugs (antiplatelet agents/ } \\
\text { NSAIDs) })^{5} / \text { Alkohol abuse }\end{array}$ & $2(10.52 \%) / 0(0 \%)$ & $0.11^{* *}$ & $* * *$ \\
\hline Diabetes mellitus & $10(52.63 \%) / 14(37.83 \%)$ & 0.56 & $1.825(0.596-5.589) ; 0.29$ \\
\hline Female sex & $12(73.68 \%) / 24(64.86 \%)$ & 0.87 & $0.929(0.294-2.935) ; 0.89$ \\
\hline \multicolumn{4}{|l|}{ Implantation: } \\
\hline TF-AVI+TSC-AVI & $15(78.94 \%) / 27(72.97 \%)$ & 0.25 & $0.720(0.192-2.696) ; 0.62$ \\
\hline TA-AVI & $4(21.05 \%) / 10(27.02 \%)$ & & \\
\hline
\end{tabular}

*Serum creatinine $\geq 200 \mu \mathrm{mol} / \mathrm{L}$ or GFR $<60 \mathrm{~mL} / \mathrm{min} / 1.73 \mathrm{~m}^{2} ;{ }^{+}$Chronic hepatic disease or bilirubin $>2 \times$ upper limit of normal, in association with aspartate aminotransferase/alanine aminotransferase/alkaline phosphatase $>3 \times$ upper limit normal; ${ }^{\ddagger} \mathrm{History}$ of anemia and/or $\mathrm{Hgb}<12.0 \mathrm{~g} / \mathrm{dL}$ on day before the procedure; ${ }^{5}$ Drugs: described separately; ${ }^{* *}$ According to Fisher exact test; ${ }^{* * *}$ Because of a small numer of patients results are not reliable; TIA - transient ischemic attack; INR - international normalized ratio; TF-AVI - transfemoral aortic valve implantation; TSC-AVI — transsubclavian aortic valve implantation; TA-AVI — transapical aortic-valve implantation; NSAIDs — nonsteroid antiinflamatory drugs

lated to the vascular complications. The number of vascular complications in the first and second year since the introduction of the procedure in our center was not significantly different and therefore was not related to the learning curve. The high proportion of reported serious bleeding and vascular complication is a result of a cumulative assessment of severe and moderate bleedings due to small participants number. In fact, major bleedings occurred between $8-15 \%$ of patients according to the used bleeding scales. Moreover vascular complications were not divided according to their severity. Similarly to our analysis, other authors report that early, major bleeding complications after TAVI affect between $9 \%$ and $31 \%$ of patients [12-17]. In a single center analysis Nuis et al. [12] proved that severe bleeding occured in $31 \%$ of patients and was the most frequent complication in the early post-procedural period. In the PARTNER study early, serious bleedings were registered in $9 \%$ to $16 \%$ of patients 
Table 6. The univariate analysis of impact of antithrombotic therapy pre-TAVI on serious bleeding according to the TIMI and GUSTO scale, in comparison with VKA monotherapy $(n=5)$.

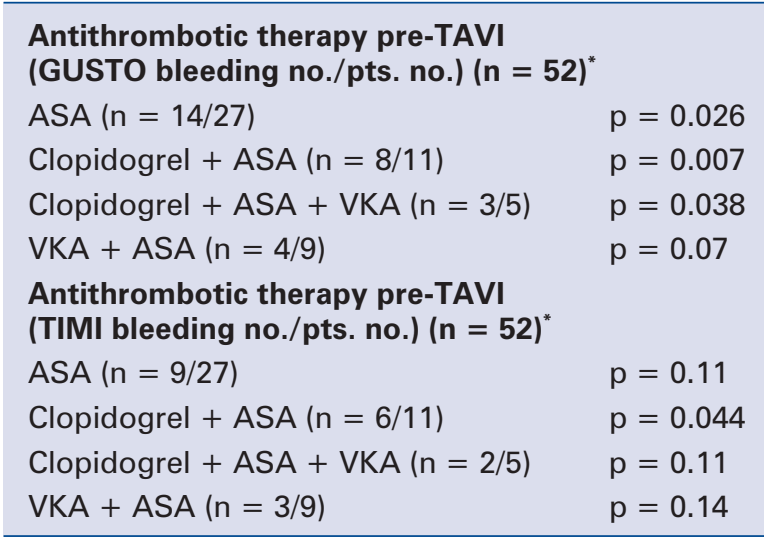

"Four patients without antithrombotic therapy; VKA — vitamin K antagonists; ASA — acetylsalicylic acid

Table 7. Bleeding complications dependent on antithrombotic therapy after TAVI in comparison with VKA + clopidogrel $(n=4)$.

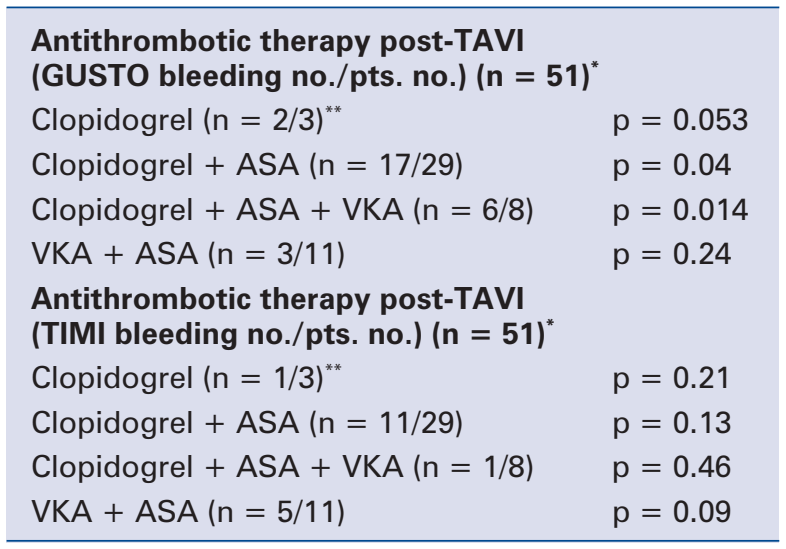

"One early death due to disruption of aorta;" high risk of bleeding; VKA — vitamin K antagonists; ASA - acetylsalicylic acid

referred for the procedure. A high proportion of bleeding was also observed at 1 year after the procedure (14.7-22.3\%) [16, 17]. In a meta-analysis of 12 studies analyzing prognostic influence of bleeding after TAVI Moreno et al. [15] demonstrated that early bleeding and vascular complications are responsible for almost $18 \%$ of deaths in the first 30 days after the procedure. In the study on 150 patients Nuis et al. [12] noted that $16 \%$ of early vascular complications were strongly related to bleeding events. Results of SOURCE registry demonstrated that vascular complications are observed in $22.9 \%$ of patients significantly increasing shortterm mortality after transapical implantation [13].
Early vascular complications affect up to $34 \%$ of patients undergoing the procedure and lead to 2 - to 3 -fold increase of the risk of 30-day mortality after TAVI $[12,18]$. The most probable cause is antiplatelet and antithrombotic treatment used before the procedure as well as treatment started after the procedure. Moreover, advanced age and comorbidities present in this population put it at high risk of frequent bleeding complications. No study analyzing the relation between anticoagulation and bleeding after TAVI has been published so far. This is particularly important as the scheme of DAPT was proposed as a standard of treatment before conducion of studies on this population of patients [2]. Most of the reports regarding the risk of combined therapy with DAPT comes from the studies on patients with acute coronary syndrome [3, 19-22]. It is known that bleeding complications are observed in $2-8 \%$ of patients with coronary artery disease and are more frequent in women, in elderly, in patients with renal dysfunction and those with a history of bleeding [3-5]. Many studies confirmed that the use of DAPT with oral anticoagulants efficiently reduces the risk of recurrent coronary incidents, but is related to worse prognosis due to increased risk of bleeding [19-22]. Chronic use of triple anticoagulant therapy (TAT) in population of patients $>75$ years of age is related to $15 \%$ risk of bleeding complications per each year of treatment [22]. In a retrospective analysis Karjalainen et al. [19] found that TAT is related to higher frequency of bleeding complications (11.4\%) and worse 1-year prognosis. Similarly Manzano-Fernández et al. [21] confirmed that TAT is an independent risk factor of bleeding in long-term observation. On the contrary, a large GRACE registry demonstrated that TAT is related to only $6 \%$ of severe bleding in the early post-procedural period [20]. Reports on the surgical procedure in patients on DAPT also demonstrate that bleeding occurs in several percent of patients [23-25]. Therefore according to EACTS recommendations antiplatelet treatment with clopidogrel should be interrupted at least 5 days before the procedure depending on the risk of bleeding and thromboembolic incidents [23, 24]. Our study demonstrated that the safest therapy consists of VKA before the procedure and a combined therapy with VKA and clopidogrel after TAVI. VKA is recommended after surgical valve replacement (SVR) $[1,26]$. This type of treatment is related to bleeding complications of 1 to over $5 \%$ /year depending on the type of the prosthesis and the clinical characteristics of patients [26]. It is proved that the maintenance of therapeutic INR values reduces the number of 
bleeding complications [23, 27]. In a retrospective analysis Karjalainen et al. [19] suggested that the safest therapy in patients undergoing percutaneous coronary interventions (PCI) with high risk of bleeding consists of the combination of VKA and clopidgrel. Monotherapy with VKA after 1 year of combined treatment is also considered as the safest one in patients with AF after PCI [21, 22]. We have no experience with VKA monotherapy after the procedure because the standard of antithrombotic treatment after TAVI consists of antiplatelet drugs with VKA added in patients with AF. Our results underline the value of monitored VKA monotherapy before and with clopidogrel after the procedure. Therefore we believe that VKA monotherapy should be considered as standard of care after TAVI with clopidogrel added only in patients after stent implantation in the preceeding 12 months. Studies on larger populations of patients undergoing TAVI are needed. To search for other causes of severe bleeding complications after TAVI established risk factors of bleeding complications were analyzed [3, 28-32]. As there is no specific scale to assess the risk of bleeding complications in the population of patients undergoing TAVI the HASBLED scale has been chosen [28]. All elements of the HASBLED scale were included in the analysis. The scale is dedicated to assess the risk of bleeding complications in patients with AF. TAVI population is a high risk population with almost all patients scoring 1 point for age over 65 years and with the presence of many comorbidities. Therefore, it was impossible to confirm the relation between high risk of bleeding in the HASBLED scale and the occurrence of severe bleeding complications. Univariate analysis of risk factors included in the HASBLED scale showed that the history of anemia and/or HGBc $<12.0 \mathrm{~g} / \mathrm{dL}$ determined during 24 hours preceeding TAVI were the strongest independent predictors of bleeding. None of the other established risk factors increased the frequency of severe bleeding complications after TAVI. This is concurent with the results of many studies which confirmed that anemia significantly increases the risk of bleeding complications and is an independent risk factor of early and long-term mortality in patients undergoing PCI or SVR [3, 28-32]. Cladellas et al. [30] demonstrated that $\mathrm{HGBc}<12.0 \mathrm{~g} / \mathrm{dL}$ before SVR is an independent risk factor of in-hospital mortality. Anemia is also a prognostic factor in heart failure [3, 31]. Our resluts are consistent with the latest reports form Halliday et al. [33] and van Mieghem et al. [34], authors discovered that near- ly $50 \%$ of patiens had anemia before TAVI, which was an independent predictor of in-hospital serious bleedings after the procedure and long-term mortality. Although $50 \%$ of patients in our analysis had anemia diagnosed on a day before TAVI we had not menaged to investigate properly the causation of it, mainly because of observational and retrospective character of our study. Possible reasons are advanced age of TAVI patients and coexistence of chronic renal impariment, which afected almost $60 \%$ of analyzed population. On the other hand, more than $90 \%$ of our patients had chronic use of many types of antithrombotic therapy before the procedure which could lead to no overt bleeding in past. The occurrence of Heyde syndrome in patients with aortic stenosis is also commonly known. Additional aim of the study was to validate the utility and prognostic value of bleeding scales in the classification of bleedings after TAVI. Although VARC had already created scale for bleeding classification after TAVI [9], we decided to use two known scales (TIMI and GUSTO) mainly due to their different criteria for bleedings classification [35, 36]. The TIMI scale is based on laboratory changes and GUSTO scale is based on clinical outcomes and blood transfusions. The need of blood transfusion which is an independent, negative predictive factor was also analyzed [6]. Moderate and severe GUSTO bleeding events occurred in over $50 \%$ of patients and major and minor TIMI bleedings were found in over $30 \%$ of patients. The effort to compare the prognostic value of both scales has been made. Moderate and severe the GUSTO bleeding was related to more frequent incidents of blood transfusion in comparison to the TIMI bleeding. This finding is concurent with the observations of Rao et al. [35] who based on the meta-analysis suggested superiority of GUSTO scale over TIMI scale in terms of sensitivity, specificity and predicitive value of bleeding. The VARC bleeding scale seems to be a combination of these two abovementioned [9] and in our further analysis, on a larger group of participants we will evaluate its prognostic value.

Although it was proved that early, post-TAVI bleeding and blood transfusions increased in-hospital mortality $[9,13,15,33,34]$, we could not confirm this findings in our analysis. The main limitation of our study was small number of participants and observational character of partially retrospective analysis, but further studies of pre-procedural anemia and impact on antithrombotic therapy on bleeding complications on a larger patients number is extremely required. 


\section{Conclusions}

1. History of anemia is the strongest predictor of serious bleeding in the GUSTO scale.

2. Oral anticoagulants used in monotherapy before TAVI and in combination with clopidogrel after the procedure are safer than antiplatelet therapy and triple drug treatment.

3. The TIMI and GUSTO scales can adequately classify bleeding after TAVI however the GUSTO scale is a better predictor of blood transfusion.

\section{Conflict of interests: none declared}

\section{References}

1. Vahanian A, Baumgartner H, Bax J et al. Guidelines on the management of valvular heart disease The Task Force on the Management of Valvular Heart Disease of the European Society of Cardiology. Eur Heart J, 2007; 28: 230-268.

2. Vahanian A, Alfieri O, Al-Attar $\mathrm{N}$ et al. Transcatheter valve implantation for patients with aortic stenosis: a position statement from the European Association of Cardio-Thoracic Surgery (EACTS) and the European Society of Cardiology (ESC), in collaboration with the European Association of Percutaneous Cardiovascular Interventions (EAPCI). Eur Heart J, 2008; 29: 1463-1470.

3. Bassand JP, Hamm CW, Ardissino D et al. Guidelines for the diagnosis and treatment of non-ST-segment elevation acute coronary syndromes. Task Force for Diagnosis and Treatment of Non-ST-Segment Elevation Acute Coronary Syndromes of European Society of Cardiology. Eur Heart J, 2007; 28: 1598-660.

4. Moscucci M, Fox KA, Cannon CP et al. Predictors of major bleeding in acute coronary syndromes: The Global Registry of Acute Coronary Events (GRACE). Eur Heart J, 2003; 24: 1815-1823.

5. Mehran R, Pocock SJ, Stone GW et al. Associations of major bleeding and myocardial infarction with the incidence and timing of mortality in patients presenting with non-ST-elevation acute coronary syndromes: A risk model from the ACUITY trial. Eur Heart J, 2009; 30: 1457-1466.

6. Shishehbor MH, Madhwal S, Rajagopal V et al. Impact of blood transfusion on short- and long-term mortality in patients with ST-segment elevation myocardial infarction. J Am Coll Cardiol Cardiovasc Interv, 2009; 2: 46-53.

7. Antman EM, Morrow DA, McCabe $\mathrm{CH}$ et al. Enoxaparin versus unfractionated heparin as antithrombin therapy in patients receiving fibrinolysis for ST-elevation myocardial infarction. Design and rationale for the Enoxaparin and Thrombolysis Reperfusion for Acute Myocardial Infarction Treatment-Thrombolysis In Myocardial Infarction study 25 (ExTRACT-TIMI 25). Am Heart J, 2005; 149: 217-226.

8. The GUSTO Investigators. An international randomized trial comparing four thrombolytic strategies for acute myocardial infarction. N Engl J Med, 1993; 329: 673-682.

9. Leon M, Piazza N, Nikolsky E et al. Standardized endpoint definitions for transcatheter aortic valve implantation clinical trials: A consensus report from the Valve Academic Research Consortium. Eur Heart J, 2011; 32: 205-217.

10. Rosenhek R, Iung B, Tornos P et al. ESC Working Group on Valvular Heart Disease Position Paper: assessing the risk of interventions in patients with valvular heart disease. doi:10.1093/eurheartj/ehr061. http://eurheartj. oxfordjournals.org/content/early//2011/03/14/eurheartj.ehr061.full.pdf + html (15 March 2011).

11. Osswald BR, Gegouskov V, Badowski-Zyla D et al. Overestimation of aortic valve replacement risk by EuroSCORE: Implications for percutaneous valve replacement. Eur Heart J, 2009; 30: 74-80.

12. Nuis RJ, Piazza N, Van Mieghem N, Otten AM et al. In-Hospital Complications After Transcatheter Aortic Valve Implantation Revisited According to the Valve Academic Research Consortium Definitions. Catheter Cardiovasc Interv. 2011;1: 457-467.

13. Thomas M, Schymik G, Walther T et al. Thirty-day results of the SAPIEN aortic Bioprosthesis European Outcome (SOURCE) Registry: A European registry of transcatheter aortic valve implantation using the Edwards SAPIEN valve. Circulation, 2010; 122: 62-69.
14. Gurvitch R, Toggweiler S, Willson $\mathrm{AB}$ et al. Outcomes and complications of transcatheter aortic valve replacement using a balloon expandable valve according to the Valve Academic Research Consortium (VARC) guidelines. EuroIntervention, 2011; 7: 41-48.

15. Moreno R, Calvo L, Salinas P et al. Causes-peri-operative-mortality-aftertranscatheter-aortic-valve-implantation-pooled-analys. J Invasive Cardiol, 2011; 23: 180-184.

16. Leon MB, Smith CR, Mack MJ et al. Transcatheter aortic-valve implantation for aortic stenosis in patients who cannot undergo surgery. N Engl J Med, 2010; 363: 1597-1607.

17. Smith CR, Leon MB, Mack MJ et al. Transcatheter versus Surgical AorticValve Replacement in High-Risk Patients. N Engl J Med, 2011; 364: 2187 -2198 .

18. Ducrocq G, Francis F, Serfaty JM et al. Vascular complications of transfemoral aortic valve implantation with the Edwards SAPIEN prosthesis: Incidence and impact on outcome. EuroIntervention, 2010; 5: 666-672.

19. Karjalainen PP, Vikman S, Niemela M et al. Safety of percutaneous coronary intervention during uninterrupted oral anticoagulant treatment. Eur Heart J, 2008; 29: 1001-1010.

20. Nguyen MC, Lim YL, Walton A et al. Combining warfarin and antiplatelet therapy after coronary stenting in the Global Registry of Acute Coronary Events: is it safe and effective to use just one antiplatelet agent? Eur Heart J, 2007; 28: 1717-1722.

21. Manzano-Fernández S, Pastor FJ, Marin F et al. Increased major bleeding complications related to triple antithrombotic therapy usage in patients with atrial fibrillation undergoing percutaneous coronary artery stenting. Chest, 2008; 134: 559-567.

22. Shireman TI, Howard PA, Kresowik TF, Ellerbeck EF. Combined anticoagulant-antiplatelet use and major bleeding events in elderly atrial fibrillation patients. Stroke, 2004; 35: 2362-2367.

23. Dunning J, Versteeg M, Fabbr A et al. Guideline on antiplatelet and anticoagulation management in cardiac surgery. Eur J Cardio-Thorac Surg, 2008; 34: 73-79.

24. Fitchett D, Eikelboom J, Fremes S et al. Dual antiplatelet therapy in patients requiring urgent coronary artery bypass grafting surgery: A position statement of the Canadian Cardiovascular Society. Can J Cardiol, 2009; 25: 683-689.

25. Ebrahimi R, Dyke C, Mehran R et al. Outcomes following pre-operative clopidogrel administration in patients with acute coronary syndromes undergoing coronary artery bypass surgery: The ACUITY (Acute Catheterization and Urgent Intervention Triage strategY) trial. J Am Coll Cardiol, 2009; 53: 1965-1972.

26. Shahbudin H. Rahimtoola. Choice of prosthetic heart valve for adult patients. J Am Coll Cardiol, 2003; 41: 893-904.

27. Ansell J, Hirsh J, Poller L, Bussey H, Jacobson A, Hylek E. The Pharmacology and management of the vitamin $\mathrm{K}$ antagonists: The Seventh ACCP Conference on Antithrombotic and Thrombolytic Therapy. Chest, 2004; 126: 204-233.

28. Pisters R, Lane DA, Nieuwlaat R, de Vos CB, Crijns HJ, Lip GY. A Novel User-Friendly Score (HAS-BLED) To Assess 1-Year Risk of Major Bleeding in Patients With Atrial Fibrillation The Euro Heart Survey. Chest, 2010; 138: 1093-1100.

29. Mehran R, Pocock SJ, Nikolsky E et al. A risk score to predict bleeding in patients with acute coronary syndromes. J Am Coll Cardiol, 2010; 55: 2556-2566.

30. Cladellas M, Bruguera J, Comin J et al. Is pre-operative anaemia a risk marker for in-hospital mortality and morbidity after valve replacement? Eur Heart J, 2006; 27: 1093-1099.

31. Ezekowitz JA, Mc Alister FA, Armstrong PW. Anemia is common in heart failure and is associated with poor outcomes: Insights from a cohort of 12065 patients with new-onset heart failure. Circulation, 2003; 107: 223-225.

32. Nikolsky E, Mehran R, Dangas G et al. Development and validation of a prognostic risk score for major bleeding in patients undergoing precutaneous coronary intervention via the femoral approach. Eur Heart J, 2007; 28:1936-1945.

33. Halliday BP, Dworakowski R, Brickham B et al. Usefulness of periprocedural bleeding to predict outcome after transcatheter aortic valve implantation. Am J Cardiol, 2012; 109: 724-728.

34. Van Mieghem NM, Nuis RJ, Tzikas A et al. Prevalence and prognostic implications of baseline anaemia in patients undergoing transcatheter aortic valve implantation. Eurointervention, 2011; 7: 184-191.

35. Rao S, O'Grady K, Pieper KS et al. A comparison of the clinical impact of bleeding measured by two different classifications among patients with acute coronary syndromes. J Am Coll Cardiol, 2006; 47: 809-816.

36. Schulman S, Angeras U, Bergqvist D, Eriksson B, Lassen MR, Fisher W. Definition of major bleeding in clinical investigations of antihemostatic medicinal products in surgical patients. J Thromb Haemost, 2010; 8: 202-204. 